\title{
Correlação entre dois testes e a pontuação do Child Health Assessment Questionnaire em crianças com cardiopatia reumática
}

\author{
Correlation between two tests and Children \\ Health Assessment Questionnaire in child with \\ rheumatic cardiopathy
}

\section{Fernanda Souza Gonçalves ${ }^{1}$ (i) Suzana Souza Moreira de Almeida² (1) Juliana Costa Santos ${ }^{3}$ Carlos Maurício Cardeal Mendes ${ }^{4}$ (])}

\begin{abstract}
'Autora para correspondência. Hospital Martagão Gesteira (Salvador). Bahia, Brasil. fernandasgfisio@gmail.com ${ }^{2}$ Hospital Ana Nery (Salvador), Hospital Martagão Gesteira (Salvador). Bahia, Brasil. suzanamoreira.fisioterapia@gmail.com ${ }^{3}$ Escola Bahiana de Medicina e Saúde Pública (Salvador), Universidade Federal da Bahia (Salvador). Bahia, Brasil. julianasantos@bahiana.edu.br
\end{abstract} 4Universidade Federal da Bahia (Salvador). Bahia, Brasil. mcardeal@ufba.br

RESUMO | INTRODUÇÃO: As crianças que evoluem para cardiopatia reumática passam a conviver com limitações, tornam-se comumente restritas no desempenho de atividade do cotidiano. OBJETIVO: Verificar a existência de correlação entre o desempenho no teste de caminhada de seis minutos (TC6M) e do degrau de três minutos (TD3) e a pontuação do child health assessment questionnaire (CHAQ) em criança com febre reumática. MÉTODOS: Estudo seccional sem grupo de comparação, descritivo e exploratório, realizado com 15 crianças. Foram avaliadas através dos testes submáximos, o TC6M e o TD3 e a avaliação da capacidade física sob a percepção do cuidador foi realizada por meio da aplicação do CHAQ. RESULTADOS: A média de idade 13,7 anos, desvio padrão 1,9 anos. A maioria dos acompanhantes eram mães, $66,7 \%$, e uma parcela considerável dos cuidadores apresentou baixo nível socioeconômico e de escolaridade. $\mathrm{O}$ valor obtido na distância percorrida através do TC6M demonstrou um baixo desempenho, mediana (intervalo interquartil) 420 (101) metros que também foi observado no teste do degrau de três minutos, mediana (intervalo interquartil) 68 (6,5) degraus. Em relação ao questionário CHAQ foi observado ausência de comprometimento da capacidade física diante da percepção dos responsáveis com escore final do questionário de 0,1. A correlação entre o questionário e o teste de caminhada de seis minutos (correlação de Sperman $=-0,21$ ) e, entre o questionário e o teste do degrau de três minutos, (correlação de Sperman = - 0,39). CONCLUSÃO: Não foi encontrada correlação dos testes submáximos, em relação à capacidade física obtida através da percepção dos responsáveis avaliada através do questionário. Esse resultado chama a atenção para a importância do fisioterapeuta inserir na sua prática clínica, nesse perfil de pacientes os testes submáximos.
ABSTRACT | INTRODUCTION: Children who develop rheumatic heart disease live with limitations, they become commonly restricted in the performance of everyday activities. OBJECTIVE: To verify the correlation between performance between two submaximal exercise tests and the questionnaire score in children with chronic rheumatic heart disease. METHODS: A cross-sectional, descriptive and exploratory study with 15 children. They were evaluated through the submaximal tests, the 6MWT and TD3 and the physical capacity assessment under the caregiver's perception was performed through the application of the questionnaire of CHAQ. RESULTS: Mean age 13.7 years, standard deviation 1.9 years. Most of the companions were mothers, $66.7 \%$, and a considerable portion of the caregivers had low socioeconomic and educational levels. The value obtained in the distance covered by the 6MWT demonstrated a low performance, median (interquartile range) 420 (101) meters, which was also observed in the three-minute step test, median (interquartile range) 68 (6.5) steps. Regarding the CHAQ questionnaire, there was an absence of impairment of physical capacity in view of the perception of those responsible with a final score of 0.1 . The correlation between the questionnaire and the six-minute walk test (Sperman correlation $=-0.21$ ) and between the questionnaire and the three-minute step test (Sperman's correlation $=-0.39$ ). CONCLUSION: No correlation was found between submaximal tests, in relation to the physical capacity obtained through the perception of those responsible, assessed through the questionnaire. This result draws attention to the importance of the physiotherapist inserting submaximal tests in his clinical practice.

KEYWORDS: Rheumatic fever. Exercise test. Activities of Daily Living. 


\section{Introdução}

A cardiopatia reumática crônica (CRC) consiste em uma complicação não supurativa da febre reumática (FR), com acometimento uni ou multivalvar, que pode vir a desencadear insuficiência cardíaca grave ${ }^{1}$. Por falta de orientação adequada, essas crianças tornamse comumente restritas, no desempenho de atividades básicas do cotidiano².

O desempenho nas atividades do cotidiano é determinado pela integração de diversas capacidades e habilidades físicas, sendo os testes físicos utilizados como ferramentas essenciais para determinação do perfil funcional, pois, além de permitirem a predição de possíveis alterações, podem ser utilizados para a avaliação do efeito das intervenções ${ }^{3,4}$. Desta maneira, a avaliação realizada de maneira adequada pelo fisioterapeuta tem como objetivo identificar limitações funcionais, bem como quantificar o reflexo da doença sobre as atividades da vida diária ${ }^{3}$.

A mensuração da funcionalidade através de alguns testes faz parte da avaliação da criança, já que eles refletem as atividades diárias, que são realizadas em níveis submáximos de esforço. Desta forma, têm sido propostos os testes funcionais submáximos, os quais são baseados em atividades de vida diária e podem refletir o nível de capacidade funcional $(C F)^{5-7}$. Cabe salientar que esses testes são de grande aplicabilidade em crianças, sobretudo pelo fato de que os máximos nem sempre são viáveis, além de proporcionarem maior risco ${ }^{8}$.

Para uma adequada abordagem a essa população, é essencial que o fisioterapeuta avalie e detenha o conhecimento das características do contexto da criança, devendo-se utilizar a impressão dos pais, como informação complementar ${ }^{9}$

Uma forma de identificar, na prática clínica, os aspectos mais influenciados por determinada condição de saúde é através de questionários, que pode ser úteis para estimar a necessidade de tratamento e investigar os determinantes do processo saúde-doença, entretanto no contexto da população pediátrica essa avaliação é realizada sob a percepção do responsável ${ }^{10}$.

Considerando a importância de adquirir conhecimento sobre esses métodos de avaliação, nessa população específica, existe a necessidade de verificar a existência de correlação entre o desempenho no teste de caminhada de seis minutos e do degrau de três minutos e a pontuação do child health assessment questionnaire (CHAQ), em criança com febre reumática.

\section{Métodos}

Estudo seccional sem grupo de comparação realizado com 15 pacientes com diagnóstico de febre reumática com amostra de conveniência, acompanhados no ambulatório de cardiopediatria de um hospital de referência de Salvador.

O estudo foi aprovado pelo Comitê de Ética em Pesquisa do Instituto de Ciências da Saúde da Universidade Federal da Bahia, denominado CEP/ICS, em 2015 com registro 395897146.6. 0000.5662. Na pesquisa foi aplicado o termo de assentimento.

Foram incluídos no estudo crianças com idade de 8 a 16 anos completos, que estavam em acompanhamento ambulatorial e que não haviam realizado nenhum procedimento cirúrgico. Todas as crianças estavam estáveis clinicamente, sem febre, estabilidade hemodinâmica, sem arritmias complexas ou potencialmente graves como fibrilação atrial/ventricular, sem marca-passos implantados. Sem limitações cognitivas, neurológicas ou ortopédicas, que compreendam as instruções para realizarem o teste de caminhada de seis minutos (TC6M) e o teste do degrau de três minutos (TD3), pais e/ou responsáveis que apresentem alguma limitação para responder o questionário.

Não foram incluídos no estudo crianças, pais e /ou responsáveis que não compreenderam as instruções para realização das avaliações propostas. 
As variáveis estudadas foram idade, sexo, escolaridade, se possui irmãos, número de pessoas por casa, tempo do diagnóstico na admissão no ambulatório, tempo de tratamento, escolaridade do responsável, renda familiar, distância caminhada no TC6M, número de degraus no TD3 e pontuação do child health assessment questionnaire (CHAQ).

Os indivíduos foram previamente avaliados pelo pesquisador e posteriormente aplicado o questionário desenvolvido pelo pesquisador e realizado os testes por um avaliador cego.

Os voluntários foram submetidos ao TC6M obedecendo aos critérios gerais padronizados pela American Thoracic Society (ATS) ${ }^{11}$, ou seja, um descanso prévio de 10 minutos no período pré-teste com medidas iniciais e finais da pressão arterial por meio do aparelho de pressão digital (TechLine $\left.{ }^{\circledR}\right)$, medidas da frequência cardíaca $(F C)$, saturação periférica de oxigênio (SpO2) pelo oxímetro de pulso (Contec ${ }^{\circledR}$ ) e sensação subjetiva de esforço ( escala de Borg de 0 - 10 pontos) foram aferidos antes, durante e depois do teste.

O TC6M foi realizado em um corredor plano com 15 metros, devidamente demarcado a cada três metros e cada volta foi considerada completa ao totalizar 30 metros (ida e volta).

A capacidade funcional foi avaliada pela distância percorrida total no fim de cada TC6, a previsão da distância percorrida foi obtida através de duas equações de referências brasileiras que são utilizadas para indivíduos saudáveis, uma referência à faixa etária é de 6 a 12 anos DTC6 $m=145,343+(11,78 \times$ idade anos $)+$ $(292,22 \times$ estatura $\mathrm{m})+(0,611 \times$ diferença absoluta na FC) - (2,684 x peso kg) 11 e a outra compreendendo a faixa etária de 13 a 84 anos de Iwana et al., 2009: DTC6 $m=622,461-(1,846 x$ idade anos $)+(61,503 x$ Gênero homens $=1$; mulheres $=0)^{13,14}$.

Após 30 minutos, a criança foi submetida ao TD3, o degrau utilizado foi construído em madeira resistente com cobertura de material antiderrapante, nas seguintes dimensões: $15 \mathrm{~cm}$ de altura por $40 \mathrm{~cm}$ de profundidade e $60 \mathrm{~cm}$ de largura. A criança deveria realizar 30 passos por minuto ${ }^{6,7}$.
A criança foi orientada a subir e descer o degrau por três minutos, objetivando o maior número possível de degraus a cada minuto (cadência livre), podendo intercalar os membros inferiores (MMII), sem o apoio dos membros superiores, os quais permaneceram estacionários ao longo do corpo. Para as análises, utilizou-se somente o desempenho no teste (número de subidas no degrau). Os sinais vitais foram aferidos seguindo a mesma avaliação do TC6M.

A avaliação da percepção dos pais em relação a funcionalidade da criança foi realizada por meio da aplicação do CHAQ - Childhood Health Assessment Questionaire, versão destinada aos pais ${ }^{15}$ adaptada e válida ${ }^{16,17}$ que mensura a capacidade funcional e independência em oito atividades da vida diária, estimando-se o grau de dificuldade ou limitações atribuídas à doença numa escala de $0-3$, sendo os maiores valores indicativos de menor capacidade.

No presente estudo, não foram calculadas estatísticas inferenciais (teste estatístico ou intervalo de confiança) dada impossibilidade da obtenção de uma estimativa confiável do erro-padrão, uma vez que o plano amostral foi não probabilístico, sendo os pacientes incluídos no estudo segundo critérios de conveniência do pesquisador, critérios de agendamento de consultas próprios do ambulatório e critérios de aceitação e elegibilidade dos pacientes, não havendo assim qualquer aleatorização no processo de seleção dos mesmos ${ }^{18,19}$.

\section{Resultados}

A amostra foi composta por quinze pacientes, dos quais a maioria foi do sexo feminino $(53,3 \%)$, com média de idade de 13,7 anos, desvio padrão (DP) 1,9, o índice de massa corporal (IMC) das crianças teve mediana (Intervalo interquartílico - IIQ) $18(3,3) \mathrm{kg} / \mathrm{m} 2$.

As crianças chegaram ao ambulatório com tempo de diagnóstico mediano de seis meses (IIQ $=6$ meses), apresentando uma mediana (IIQ) 3,7 (3) anos de acompanhamento até o período da coleta e todas elas fazendo uso de benzetacil, a cada 21 dias (Tabela 1). 
Tabela 1. Características clínico-epidemiológicas das crianças com cardiopatia reumática, Salvador, BA, 2015 (N=15)

\begin{tabular}{|c|c|c|c|}
\hline Variáveis & n (\%) & $\begin{array}{c}\text { Promédio } \\
\text { (Variabilidade) }\end{array}$ & Mínimo; máximo \\
\hline \multicolumn{4}{|l|}{ Sexo } \\
\hline Masculino & $7(46,7)$ & - & - \\
\hline Feminino & $8(53,3)$ & - & - \\
\hline Idade (anos) $)^{\star \star}$ & - & $13,7(1,9)$ & 10,$6 ; 16,8$ \\
\hline Peso $(\mathrm{Kg})^{*}$ & - & $42(10,6)$ & - \\
\hline Altura $(m)^{\star \star}$ & - & $1,6(0,1)$ & 1,$4 ; 1,6$ \\
\hline \multicolumn{4}{|l|}{ Escolaridade } \\
\hline Frequenta & $15(100 \%)$ & - & - \\
\hline Não frequenta & 0 & - & - \\
\hline Possuem irmãos & $15(100 \%)$ & - & - \\
\hline Pessoas por casa** & - & $4(1,6)$ & $2 ; 8$ \\
\hline $\begin{array}{l}\text { Tempo diagnóstico } \\
\text { na admissão/ } \\
\text { meses* }\end{array}$ & - & $6(6)$ & - \\
\hline $\begin{array}{l}\text { Tempo de } \\
\text { tratamento até a } \\
\text { coleta/ anos* }\end{array}$ & - & $3,7(3)$ & - \\
\hline
\end{tabular}

Em relação aos responsáveis que acompanharam a criança na consulta ambulatorial, a maior parte da amostra foi constituída por mães $66,7 \%$ e as famílias apresentavam baixo nível socioeconômico com predomínio de renda salarial de até um salário mínimo 73,3\% (Tabela 2).

Tabela 2. Características dos responsáveis das crianças com cardiopatia reumática. Salvador, BA, 2015 (N=15)

\begin{tabular}{lc}
\hline Variáveis & $\mathbf{n}(\%)$ \\
\hline Responsável & $10(66.7 \%)$ \\
Mãe & $3(20 \%)$ \\
Pai & $2(13,3 \%)$ \\
Outros & \\
Escolaridade do responsável & $2(13,3 \%)$ \\
Analfabeto & $10(66,7 \%)$ \\
$1^{\circ}$ completo/ incompleto & $3(20 \%)$ \\
$2^{\circ}$ completo/ incompleto & 0 \\
Superior & \\
Renda por salário mínimo & $1(6,7 \%)$ \\
Menos de 1 salário & $11(73,3 \%)$ \\
Até 1 salário & $3(20 \%)$ \\
Até 2 salários & \\
\hline
\end{tabular}

Todos os pacientes foram avaliados pelo TC6M e o valor obtido na distância percorrida demonstrou um baixo desempenho, mediana (IIQ) 420 (101) metros. Já a distância percorrida prevista, obtida pela equação de referência para população saudável, apresentou mediana (IIQ) $592(79,2)$ metros. (Figura 1). Entretanto, três crianças interromperam o teste antes de completar os seis minutos, relatando fadiga em MMII (Borg 7- Muito intenso) e nenhuma criança retornou ao teste após a interrupção.

Todas as crianças realizaram o TD3, o valor obtido do total de subidas e descidas em três minutos demonstrou um baixo desempenho, mediana (IIQ) 68 (6,5), entretanto, dois pacientes apresentaram valores superiores a 90 subidas e descida no final do teste. 
Figura 1. Distância percorrida e prevista no TC6M das crianças com cardiopatia reumática. Salvador, BA, 2015 (N=15)
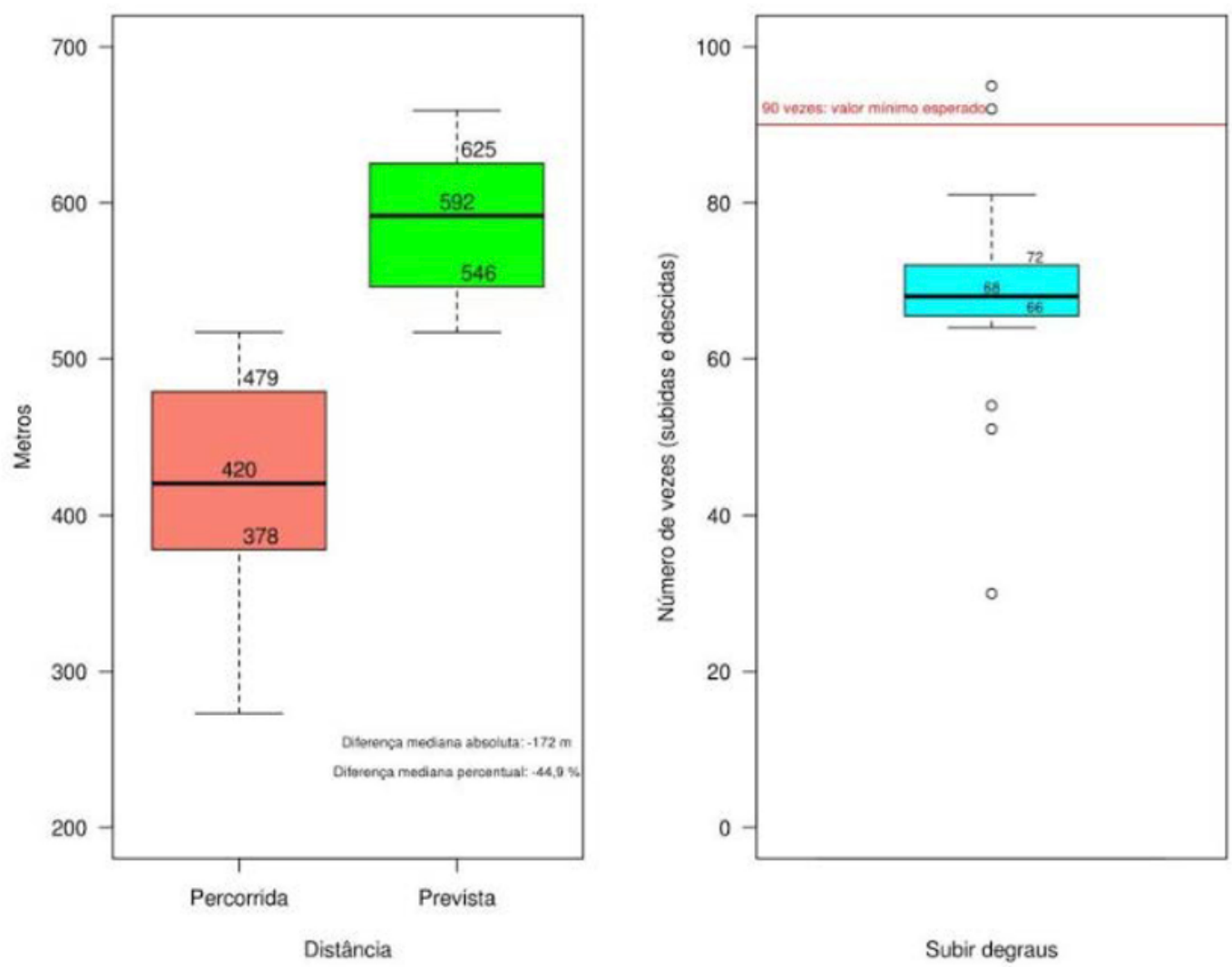

Em relação aos itens do CHAQ entre a população do estudo, nos domínios vestir-se e arrumar-se, levantar-se, alimentar-se, andar, realizar a higiene, alcançar, apanhar e atividades, todos os pacientes apresentaram, no escore final de 0,1, o que corresponde à ausência de comprometimento da capacidade física e funcional diante da percepção dos responsáveis. Entretanto, alguns responsáveis sinalizaram comprometimento de determinados domínios: levantar-se, caminhar, alcançar, preensar (apanhar) e atividades, contudo isso não refletiu nos resultados.

Figura 2. Itens do CHAQ das crianças com cardiopatia reumática. Salvador, BA, 2015 (N=15)
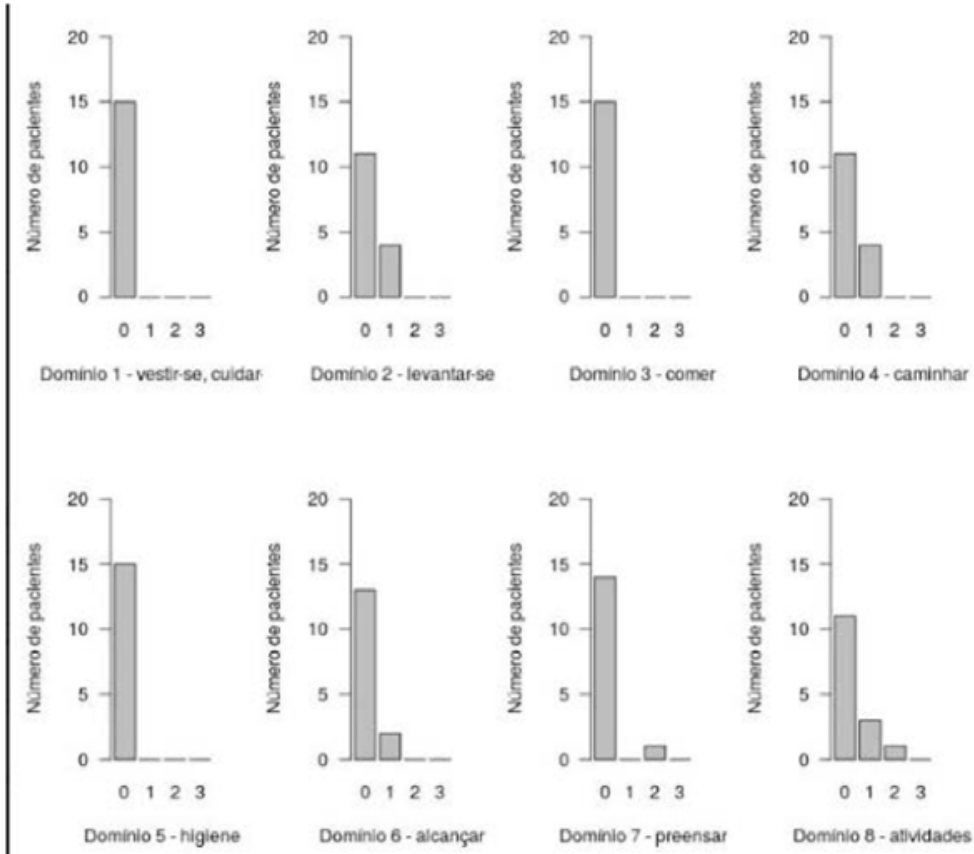

Logenda dos dominios: 0.sem nenhum dficuldade; 1-com alguma dificuldade, 2 com muita dificuldade, 3 incapaz de tazes 


\section{Discussão}

Os resultados do presente estudo demostraram uma baixa correlação entre a percepção dos cuidadores, obtido através do CHAQ e os testes submáximos, uma vez que foram atribuídos pelos cuidadores escores que indicavam uma boa condição funcional para os pacientes, ao passo que os testes submáximos demostraram que a condição física foi inferior ao esperado para os parâmetros de referência.

Alguns fatores podem, a princípio, explicar esses achados. Talvez, devido ao fato de todas as crianças estarem em acompanhamento ambulatorial, clinicamente estáveis do ponto de vista da doença e conviverem com outros irmãos saudáveis, tenha dificultado para o cuidador identificar o comprometimento funcional da criança, conforme foi visto nos resultados do questionário CHAQ. Além desses fatores, o baixo nível de escolaridade, socioeconômico e o entendimento sobre a febre reumática por parte dos cuidadores, podem ter contribuído, também, para essa condição.

Diante da outra possibilidade que pode ter refletido no resultado do $\mathrm{CHAQ}$, ressalta-se que a mãe constituiu a maior parte dos acompanhantes no nosso estudo, assumindo, também, as responsabilidades sobre o cuidar dos outros filhos. Dessa forma, a sua atenção e assistência são dissipadas e não concentradas apenas naquele que é portador da cardiopatia reumática crônica $(C R C)$, já que nenhuma criança do estudo apresentou sintomatologia que demandasse cuidados constantes. Achados semelhantes foi encontrado em um estudo, que identificaram a mãe, no contexto familiar como função essencial no cuidado com os filhos, que tem condições de definir a necessidade de cada filho e que desempenha o papel principal de cuidadora ${ }^{20}$.

Outro achado que chamou a atenção foi o aspecto da escolaridade dos responsáveis, pois pode também apresentar-se como influenciadora nos resultados, uma vez que maior nível de instrução e conhecimento possibilita uma forma diferenciada na percepção da doença e no cuidado com a saúde da criança ${ }^{21}$. É necessário ressaltar que irão existir pais ou cuidadores com baixo grau de escolaridade que cuidam meIhor de suas crianças do que outros com maior grau. Contudo, o que se objetiva refletir aqui é que, de maneira geral, um maior nível de conhecimento garante maiores chances de identificar, de maneira precoce, alterações decorrentes da doença.
Alguns autores afirmam no seu estudo que, aproximadamente, $10 \%$ de crianças com doença crônica sofrem de alguma limitação nas suas atividades diárias $^{22}$. No presente estudo, essa condição foi identificada, ao avaliar as crianças através dos testes submáximos e, a partir dos resultados, foi observado que essas crianças apresentaram um desempenho inferior ao esperado. O baixo desempenho físico foi observado em um estudo que utilizou o TC6M para avaliar tolerância ao exercício físico e resposta cardiorrespiratória. Ao realizar comparação com indivíduos saudáveis, os cardiopatas caminharam menores distâncias $\left(472,5 \mathrm{~m}\right.$ e $548,8 \mathrm{~m}$, respectivamente) ${ }^{23}$.

Outro estudo encontrou valores médios de distância percorrida, de 557 e 656, em crianças cardiopatas e saudáveis, acima de oito anos, indicando que crianças cardiopatas apresentam capacidade física reduzida, quando comparadas com populações saudáveis. A literatura sugere que o tipo de esforço durante o TC6M assemelha-se à atividade diária, uma vez que a maioria dessas atividades é realizada em nível submáximo de esforços. Acredita-se que a distância reflete melhor as atividades físicas diárias do que testes de exercício máximo ${ }^{12,24}$

Neste estudo, durante o TC6M, três crianças interromperam o teste antes de completar os seis minutos, pois relataram fadiga em MMII. Resultado semelhante foi encontrado indicando que as crianças cardiopatas, possivelmente, apresentam maior prejuízo da musculatura periférica global, portanto, mais relacionado à falta de condicionamento muscular do que à resposta respiratória ao exercício ${ }^{25}$.

Os achados sugerem que a avaliação da funcionalidade obtida por meio do TC6M, torna esse teste submáximo um instrumento seguro para avaliar a CF na população em questão. O que surpreende nos resultados encontrados é que esses pacientes, mesmo submetidos a um segmento ambulatorial por condições crônicas potencialmente fatais e/ou incapacitantes, a aplicabilidade do questionário aos cuidadores não conseguiu identificar, no dia a dia dessas crianças, o reflexo da doença que foi evidenciado pelos testes. Esses prejuízos, observados na capacidade física no TC6M, também foram identificados no TD3, pois as crianças cursaram com o desempenho inferior ao previsto, reforçando os dados encontrados no outro teste. 
Alguns autores compararam o TC6 com o TD3, como medidas de tolerância ao exercício, em 28 crianças, e os autores concluíram que o TD3 é fácil de executar e bem tolerado em criança com doença crônica grave. Diante dos resultados encontrados no presente estudo, sugere-se que o teste do degrau pode ser uma alternativa para substituir o teste de caminhada quando não houver espaço físico amplo para sua realização, porém fazem-se necessários, ainda, futuros estudos para a sua padronização. Em relação aos dois pacientes desse estudo que mostraram bom desempenho e apresentaram valores superiores a 90 subidas e descidas no fim do TD3, apesar de não terem alcançando a distância prevista no TC6M, foram os que apresentaram melhor desempenho na distância percorrida ${ }^{26}$.

Tendo em vista os resultados encontrados, no que diz respeito aos testes submáximos, foi observada uma forte correlação entre a distância percorrida e o número de subidas e descidas, significando que os indivíduos analisados apresentaram desempenhos semelhantes na avaliação dos dois testes. Esses resultados estão de acordo com o esperado, uma vez que ambos se propõem a avaliar e predizer a capacidade de exercício no desempenho das atividades de vida diária, sendo, ainda, capazes de permitir o diagnóstico precoce da limitação da atividade física.

Esses testes são considerados como formas alternativas, com o intuito de avaliar a CF, monitorização da eficácia de intervenções, especialmente como parâmetros de resposta a programas de reabilitação e seguimentos de protocolos fisioterapêuticos, além de poder direcionar o tratamento de maneira individualizada ${ }^{27,28}$.

É interessante observar que, através dos achados deste estudo, foi possível obter informações a respeito do questionário como ferramenta para auxiliar os profissionais de saúde a compreender e obter informações a respeito da percepção individual de cada cuidador, no contexto da convivência com a criança portadora de doença crônica. Foi possível constatar, também, que o grau de subjetividade de uma avaliação aumenta à medida que o sujeito investigado não é avaliado diretamente.
Dessa forma, deve-se tomar cuidado, já que, muitas vezes, na população pediátrica, as respostas pelos pais ou cuidadores são aceitas como medidas confiáveis do estado de saúde e, na prática, decisões são tomadas baseadas na opinião dos cuidadores. A limitação desse estudo é que a maioria dos pacientes deste estudo reside no interior da Bahia e o acompanhamento ambulatorial é realizado por consultas semestrais e, diante dessa condição, alguns pacientes não compareceram ao ambulatório nos dias agendados.

\section{Conclusão}

Diante dos resultados obtidos pelo questionário CHAQ, foi observado que diante da percepção dos responsáveis as crianças não possuíam comprometimento da $C F$, entretanto a redução da tolerância aos esforços foi observada através dos testes submáximos, que constataram redução da distância pecorrida através do TC6M e do número de subidas e descidas no TD3 indicando não haver uma correlação entre a percepção dos responsáveis e o que os pacientes realmente apresentam fisicamente, visto nos testes. Frente a esses resultados, sugere-se que a entrevista com os responsáveis seja utilizada de maneira complementar durante as consultas e não como forma de substituir a avaliação minuciosa e individual que deve ser realizada em cada paciente.

Apesar dos testes submáximos, muitas vezes não fazerem parte da rotina de avaliação de muitos fisioterapeutas na população pediátrica, esses teste podem refletir os efeitos da doença nas atividades de vida diária, além de serem de fácil aplicabilidade, baixo custo, bem tolerados e serve para estabelecimento de prognóstico a partir de predição de morbidade e mortalidade. Desta forma é possível estabelecer um diagnóstico funcional e instituir tratamentos direcionados para necessidade específica de cada criança/adolescente além de servir como parâmetro pré e pós-intervenção. 


\section{Contribuições dos autores}

Mendes CMC participou da concepção, delineamento, busca e análise estatística dos dados da pesquisa, interpretação dos resultados e redação do artigo científico. Carvalho FSG participou da concepção, delineamento, interpretação dos resultados e redação do artigo científico. Almeida SSM participou da concepção e participou da coleta de dados da pesquisa. Santos JC participou da concepção do estudo.

\section{Conflitos de interesses}

Nenhum conflito financeiro, legal ou político envolvendo terceiros (governo, empresas e fundações privadas, etc.) foi declarado para nenhum aspecto do trabalho submetido (incluindo, mas não se limitando a subvenções e financiamentos, participação em conselho consultivo, desenho de estudo, preparação de manuscrito, análise estatística, etc.).

\section{Referências}

1. Ladeia AMT, Gama JMA, Fernandes CR, Santos FS, Cordeiro BS, Cruz RCC. Predictors of Unfavourable Outcomes in Children and Adolescents Submitted to Surgical Mitral Valvuloplasty Secondary to Chronic Rheumatic Heart Disease. Arq Bras Cardiol. 2019;113(4):748-756. doi: 10.5935/abc.20190184

2. Kao CC, Chanq PC, Chiu CW, Wu LP, Tsai JC. Physical activity levels of schoolage child with congenital heart disease in Taiwan. Appl Nurs Res. 2009;22(3):191-7. doi: 10.1016/j.apnr.2007.12.002

3. Rogers D, Prasad SA, Doull I. Exercise testing in children with cystic fibrosis. J R Soc Med. 2003;96(43):23-29.

4. Enright PL. The six-minute walk test. Respir Care. 2003;48(8):783-5.

5. Zwiren LD. Considerações sobre testes de esforco e sua prescrição durante a infância. In: Manual de pesquisa das diretrizes do ACSM (American College Sports Medicine) para os testes de esforço e sua prescrição. 4.ed. Rio de Janeiro: Guanabara Koogan; 2003. P. 522-28.

6. Gomes ELFD, Silva DS, Costa D. Physical capacity tests in pediatrics. Fisioter. Brasil. 2012;13(6).

7. Schnaider J, Karsten M. Testes de tolerância ao exercício em programa de fisioterapia hospitalar após exacerbação da doença pulmonar obstrutiva crônica. Fisioter Mov. 2006;19(4):119-26.

8. Regamey N, Moeller A. Paediatric exercise testing. Eur Respir Mon. 2010;47:291-309. doi: 10.1183/1025448x.00013109
10. Eliser C, Morse R. Quality-of-life measures in chronic diseases of childhood. Health Technol. 2001;5(4):1-157. doi: 10.3310/ $\underline{\text { hta5040 }}$

11. ATS Committee on Proficiency Standards for Clinical Pulmonary Function Laboratories. ATS Statement: Guideline for the six-minute walk test. Am J Respir Crit Care Med. 2002;166(1):111-7. doi: 10.1164/ajrccm.166.1.at1102

12. Priesnitz CV, Rodrigues GH, Stumpf CS, Viapiana G, Cabral $\mathrm{CP}$, Stein RT et al. Reference values for the 6-min walk test in healthy children aged 6-12 years. Pediatric Pulmonology. 2009;44(12):1174-9. doi: 10.1002/ppul.21062

13. Iwama AM, Andrade GN, Shima P, Tanni SE, Godoy I, Dourado VZ. The six-minute walk test and body weight-walk distance product in healthy Brazilian subjects. Braz J Med Biol Res. 2009;42(11):1080-1085. doi: 10.1590/S0100-879X2009005000032

14. Dourado VZ. Equações de referência para o teste de caminhada de seis minutos em indivíduos saudáveis. Arq Bras Cardiol. 2011;96(6):128-138. doi: 10.1590/S0066782X2011005000024

15. Sing G, Athreya BH, Fries JF, Goldsmith DP. Measurement of health status in children with juvenile rheumatoid arthritis. Arthritis Rheum. 1994;37(12):1761-1769. doi: $10.1002 /$ art.1780371209

16. Len C, Goldenberg J, Ferraz MB, Hilario MO, Oliveira LM, Sacchetti S. Cross cultural reliability of the Childhood Health Assessment Questionnaire. J Rheumatol. 1994;21(12):2349-52.

17. Machado CSM, Ruperto N, Silva CHM, Ferriane VPL, Roscoe I, Campos LMA et al. The Brazilian version of the Childhood Health Assessment Questionnaire (CHAQ) and the Child Health Questionnaire (CHAQ). Clin Exp Rheumatol. 2001;19(23):25-29, 2001.

18. Ludwig DA. Use and Misuse of p-Values in Designed and Observational Studies: Guide for Researchers and Reviewers. Aviation, Space, and Environmental Medicine. 2005;76(7):675-680.

19. Pereira MG. Epidemiologia. Rio de Janeiro: Guanabara Koogan LTDA; 1995.

20. Padilha RD. Mediação sistêmica interativa: família e escola, construindo uma cultura de paz. Curitiba: Amanapaz; 2004.

21. Case A, Lubotsky D, Paxson C. Economic status and health in childhood: the origins of the gradient. The American Economic Review.2002;92(5):1308-1334. doi: 10.1257/000282802762024520

22. Garralda ME. Chronic physical illness and emotional disorder in childhood. Br J Psychiatry. 1994;164(1):8-10. doi: $10.1192 /$ bjp.164.1.8

9. Andrade LB. Fisioterapia respiratória em neonatologia e pediatria. São Paulo: Medbook; 2010. 
23. Moalla W, Gauthier R, Maingourd Y, Ahmaidi S. Six-minute walking test to assess exercise tolerance and cardiorespiratory responses during training program in children with congenital heart disease. Int J Sports Med.2005;26(9):756-62. doi: 10.1055/s2004-830558

24. Geiger R, Strasak A, Treml B, Gasser K, Kleinsasser A, Fischer $\checkmark$ et al. Six-minute walk test in children and adolescents. J Pediatr. 2007;150(4):395-9. doi: 10.1016/j.jpeds.2006.12.052

25. Inoue AS. Estudo do teste de caminhada de seis minutos, variabilidade da frequência cardíaca, função pulmonar e força muscular respiratória em crianças e adolescentes submetidos à correção cirúrgica de cardiopatia congênita [dissertação]. São Paulo: Faculdade de Medicina. Universidade de São Paulo; 2013.

26. Aurora P, Prasad SA, Balfour-Lynn IM, Slade G, Whitehead B, Dinwiddie R. Exercise tolerance in children with cystic fibrosis undergoing lung transplantation assessment. Eur Respir J London. 2001;18(2): 293-297. doi: 10.1183/09031936.01.00058701

27. Noonan V, Dean E. Submaximal exercise testing: clinical application. Phys Ther. 2000;80(8):78-807.

28. Solway S, Brooks D, Lacasse Y, Thomas S. A qualitative systemic overview of the measurement properties of functional walk tests used in the cardiorespiratory domain. Chest.

2001;119(1):256-270. doi: 10.1378/chest.119.1.256 